Provided for non-commercial research and education use. Not for reproduction, distribution or commercial use.

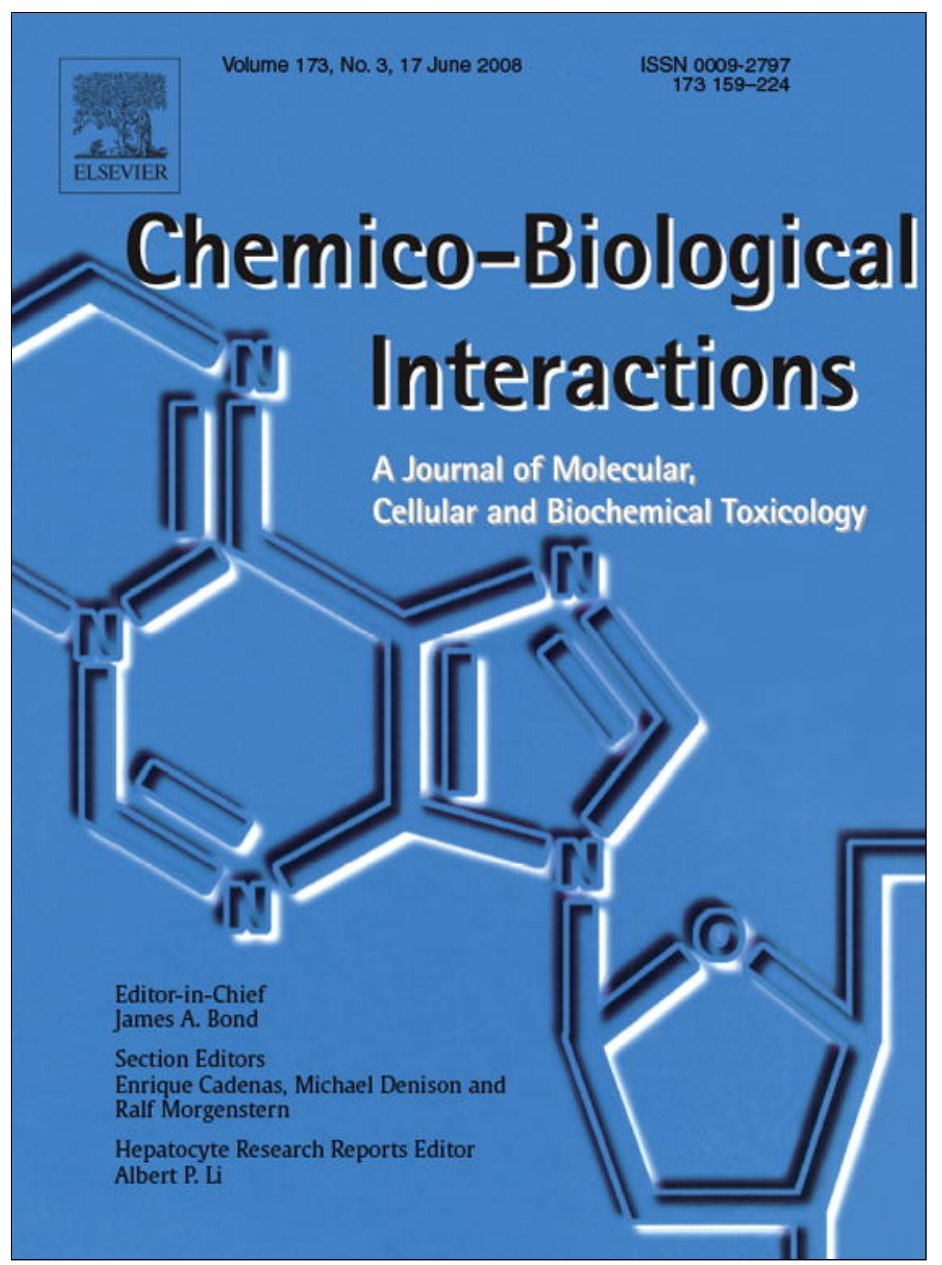

This article appeared in a journal published by Elsevier. The attached copy is furnished to the author for internal non-commercial research and education use, including for instruction at the authors institution and sharing with colleagues.

Other uses, including reproduction and distribution, or selling or licensing copies, or posting to personal, institutional or third party websites are prohibited.

In most cases authors are permitted to post their version of the article (e.g. in Word or Tex form) to their personal website or institutional repository. Authors requiring further information regarding Elsevier's archiving and manuscript policies are encouraged to visit:

http://www.elsevier.com/copyright 


\title{
Comparative effects of three 1,4-dihydropyridine derivatives [OSI-1210, OSI-1211 (etaftoron), and OSI-3802] on rat liver mitochondrial bioenergetics and on the physical properties of membrane lipid bilayers: Relevance to the length of the alkoxyl chain in positions 3 and 5 of the DHP ring
}

\author{
Maria A.S. Fernandes ${ }^{a}, *$, Susana P.S. Pereira ${ }^{b}$, Amália S. Jurado ${ }^{c}$, \\ José B.A. Custódio ${ }^{\mathrm{d}}$, Maria S. Santos ${ }^{\mathrm{e}}$, António J.M. Moreno ${ }^{\mathrm{a}}$, \\ Gunars Duburs ${ }^{\mathrm{f}}$, Joaquim A.F. Vicente ${ }^{\mathrm{g}}$ \\ a IMAR, Departamento de Zoologia, Universidade de Coimbra, 3004-517 Coimbra, Portugal \\ b Centro de Neurociências e Biologia Celular, Universidade de Coimbra, 3004-517 Coimbra, Portugal \\ c Centro de Neurociências e Biologia Celular, Departamento de Bioquímica, Universidade de Coimbra, \\ 3001-401 Coimbra, Portugal \\ d Centro de Neurociências e Biologia Celular, Faculdade de Farmácia, Universidade de Coimbra, \\ 3000-295 Coimbra, Portugal \\ e Centro de Neurociências e Biologia Celular, Departamento de Zoologia, Universidade de Coimbra, \\ 3004-517 Coimbra, Portugal \\ ${ }^{\mathrm{f}}$ Latvian Institute of Organic Synthesis, 21 Aizkraukles Street, Riga LV-1006, Latvia \\ g IMAR, Departamento de Botânica, Universidade de Coimbra, 3000-393 Coimbra, Portugal
}

\section{A R T I C L E I N F O}

\section{Article history:}

Received 1 February 2008

Received in revised form 3 March 2008

Accepted 5 March 2008

Available online 15 March 2008

\begin{abstract}
A B S T R A C T
The 1,4-dihydropyridines OSI-1210, OSI-1211 (etaftoron), and OSI-3802 are compounds with similar chemical structure. They differ by the length of the alkoxyl chain in positions 3 and 5 of the dihydropyridine (DHP) ring and by their pharmacological action characteristics. However, as far as we know, a clear relationship between the effects of these compounds and the length of the alkoxyl chain in positions 3 and 5 of the DHP has not been established. The goal of this study was to compare the influence of OSI-1210, OSI-1211 (etaftoron), and OSI-3802 on rat liver mitochondrial bioenergetics and on the physical properties of membrane lipid bilayers, correlating their actions with the length of the alkoxyl chain in positions 3 and 5 of the DHP ring. Using either glutamate/malate or succinate as respiratory substrates, all the compounds, in concentrations of up to $500 \mu \mathrm{M}$, depressed state 3 and uncoupled respiration, respiratory control (RCR) and ADP/O ratios, and phosphorylation rate, whereas state 4 respiration was stimulated. However, the stimulatory effect on state 4 induced by OSI-3802, the compound with the longest chain in positions 3 and 5 of the DHP ring, as well as its inhibitory effects on RCR and ADP/O ratios and phosphorylation rate were more pronounced than that induced by OSI-1210 and OSI-1211 (etaftoron), the compounds with the shortest and intermediate chains, respectively. Moreover, OSI-3802 maximized state 4 stimulation and minimized RCR and ADP/O ratios, and phosphorylation rate at a concentration of $100 \mu \mathrm{M}$, whereas low graduate effects were detected with OSI-1210 and OSI-1211 (etaftoron) for concentrations of up to $500 \mu \mathrm{M}$. At
\end{abstract}

\footnotetext{
* Corresponding author. Tel.: +351 239 855760; fax: +351 239855789.

E-mail address: mfer@ci.uc.pt (M.A.S. Fernandes).
} 
low concentrations $(\leq 30 \mu \mathrm{M})$, OSI-3802, like its analogue OSI-1212 (cerebrocrast), reduced the phase transition temperature, the cooperative unit size, and the enthalpy associated with the phase transition temperature of dimyristoylphosphatidylcholine (DMPC) membrane bilayers. A good correlation was established between the effects of $200 \mu \mathrm{M}$ OSI-1210, OSI-1211 (etaftoron), and OSI-3802 on glutamate/malate- and succinate-dependent RCR of rat liver mitochondria and on the enthalpy change $(\Delta H)$ for the thermotropic profile of DMPC membrane bilayers at a $0.2 \mathrm{drug} / \mathrm{DMPC}$ molar ratio, indicating that the changes induced by these compounds on both mitochondrial membrane integrity and physical properties of DMPC membrane bilayers are strongly related to the length of the alkoxyl chain in positions 3 and 5 of the DHP ring. A putative relationship between membrane physical perturbation, bioenergetics impairment and the molecular characteristics of the compounds will be established as an approach to better understand their differentiated toxicological and pharmacological actions.

(c) 2008 Elsevier Ireland Ltd. All rights reserved.

\section{Introduction}

According to the present conception "the 1,4dihydropyridine (1,4-DHP) structure is a pharmacophoric template or privileged structure that, when appropriately substituted, can exert potent and selective actions at a diverse set of membrane structures, including ion channels, G-protein-coupled receptors and enzymes" [1]. DHPs possess different pleiotropic properties [2,3]. Thus, depending on the chemical structure peculiarities 1,4-DHPs have electron/hydrogen donor, peptide-type and lipid-like characteristics and many regulatory activities: neuro- and radioprotection, anti-mutagenic, anti-diabetic, anti-inflammation, anti-ischaemic/anti-anginal and antihypertensive actions, as well as growth stimulating, life span prolongation and gene transfection properties [4]. Therefore studies of specific properties of a group of DHP derivatives comprising closely related structures could be useful for elucidation of chemical-biological interaction, particularly, influence of minor changes of substituent structure.

The 1,4-dihydropyridines OSI-1210, OSI-1211 (etaftoron), OSI-1212 (cerebrocrast), and OSI-3802, with a common chemical formula (Fig. 1), differ by the length of the alkoxyl chain in positions 3 and 5 of the DHP ring. The OSI-1210 is the compound with the shortest chain, OSI1211 (etaftoron) has an intermediate chain, while OSI-1212 (cerebrocrast) and its isomer, OSI-3802, possess the longest chain. Along to these structural differences, the compounds differ by their pharmacological action specificity and efficacy both in "in vitro" and "in vivo" studies. Thus, "in vitro" studies showed that both OSI-1211 (etaftoron) and OSI1212 (cerebrocrast), at low concentrations $\left(10^{-7}\right.$ down to $10^{-9} \mathrm{M}$ ), intensified lymphocyte proliferation in the presence of IL-2 and insulin. However, the effect of OSI-1212 (cerebrocrast) was higher than that of OSI-1211 (etaftoron) [5]. Differently, the effectiveness of OSI-1212 (cerebrocrast) to interact with glucocorticoid hormone receptors from rat liver cytosol was lower than that of OSI-1211 (etaftoron), as reflected by the high apparent $\mathrm{IC}_{50}$ value of OSI-1212 $(40.74 \mu \mathrm{M})$ to inhibit specific ligand [1,2,4(3) $\mathrm{H}$ ]-dexamethasone-glucocorticoid receptor interaction, as compared with that of OSI-1211 (23.71 $\mu \mathrm{M})$ [6]. In "in vivo" studies showed that administration of OSI-1211 (etaftoron), OSI-3802, or OSI-1212 (cerebrocrast) significantly decreased the blood glucose concentration in normal and STZ-diabetic rats after a single administration at 0.5 and $1.5 \mathrm{mg} \mathrm{kg}^{-1}$, p.o., doses. However, OSI-1212 (cerebrocrast) was the most effective compound on glucose metabolism [7]. The stronger effect of OSI-1212 (cerebrocrast), as compared with its analogues, was explained to be due to the increase in the number of carbon atoms in the ester moiety in positions 3 and 5 of the DHP structure, thereby modifying the properties of the compound [7]. However, the strict dependence on the hydrocarbon chain length cannot explain differential effects of OSI-3802 and its isomer OSI-1212 (cerebrocrast) as previously reported [7]. From another point of view, OSI-1211 (etaftoron) decreases haemoglobin affinity to oxygen [8], and is proposed for the treatment of disturbances in oxygen supply to the brain.

However, as far as we know, a clear relationship between the effects of these compounds and the length of the alkoxyl chain in positions 3 and 5 of the dihydropyridine (DHP) has not been established. As we envisage that this molecular characteristic may modulate molecules membrane interactions and, on the other hand, we are aware that such interactions are decisive in establishing the biological activity of many different compounds $[9,10]$, our study was designed to compare the effects of OSI-1210, OSI-1211 (etaftoron), and OSI-3802 on mitochondrial bioenergetics, a physiological function that depends on membrane integrity and on the physical properties of the lipid bilayer. Parallel biophysical studies were performed using multilamellar vesicles of dimyristoylphosphatidylcholine (DMPC) as a biological membrane model. Although hardly reflecting the complex structure of biomembranes, synthetic lipid bilayers have the advantage to allow an easier interpretation of drug induced membrane physical events [11].

Mitochondria were chosen as an experimental model because it is increasingly recognized that these organelles are potential targets to pharmacological and toxicological actions of membrane-active agents [12]. Also, the mitochondriotropic character of the 1,4-dihydropyridines, particularly fluorine containing ones, is well expressed and shown in many publications [13]. Thus, the calcium antagonist foridone (ryodipine, PP-1466), which possesses the same substituent in the position 4 as OSI-1212 (cerebrocrast) and its analogues studied herein, improves mitochondrial bioenergetics in the isolated rat mitochondria from the infarct zone of the heart as well as from relatively undamaged zone [14]. Moreover, we have shown OSI-1212 (cerebrocrast) ability to interact with rat skeletal, 
<smiles>[R]OCCOC(=O)C1=C(C)NC(C)=C(C(=O)OCCO[R])C1c1ccccc1OC</smiles>
1, $\mathrm{R}=\mathrm{CH}_{3}$
(OSI-1210),
2, $\mathrm{R}=\mathrm{C}_{2} \mathrm{H}_{5}$,
(OSI-1211, Etaftoron),
3, $\mathrm{R}=\mathrm{CH}\left(\mathrm{CH}_{3}\right)_{2}$, (OSI-3802),
4, $\mathrm{R}=\mathrm{CH}_{2} \mathrm{CH}_{2} \mathrm{CH}_{3}$, (OSI-1212, Cerebrocrast)

Fig. 1. Molecular structures of OSI-1210 (1), OSI-1211 (etaftoron) (2), OSI-3802 (3), and OSI-1212 (cerebrorast) (4).

neuronal and hepatic mitochondria $[15,16]$, to incorporate into mitochondrial membranes and to induce lipid membrane organization changes [17]. Mitochondria have also been considered as an essential intracellular target for OSI1212 (cerebrocrast) neuroprotective actions [18,19]. It could also be mentioned that DHP derivative ryodipine has binding constant to mitochondria rather than to microsomes [20].

The results obtained in this study are expected to be a relevant contribution to understand the importance of structural molecular details, namely the length of the alkoxyl chain in positions 3 and 5 of the DHP structure, for the biological activity of these 1,4-dihydropyridines.

\section{Materials and methods}

\subsection{Animals}

Male Wistar rats (250-350 g), housed at $22 \pm 2{ }^{\circ} \mathrm{C}$ under artificial light for 12-h light/dark cycle and with access to water and food ad libitum, were used throughout the experiments. The experiments reported here were carried out in accordance with the National Requirements for Vertebrate Animal Research and in accordance with the European Convention for the Protection of Animals used for Experimental and other Scientific Purposes.

\subsection{Isolation of rat liver mitochondria}

Rat liver mitochondria were isolated from male Wistar rats by differential centrifugation according to conventional methods [21]. After washing, the pellet was gently resuspended in the washing medium at a protein concentration of about $50 \mathrm{mg} \mathrm{ml}^{-1}$. Protein content was determined by the biuret method [22].

\subsection{Measurements of respiratory activities}

Oxygen consumption was monitored polarographically at $30^{\circ} \mathrm{C}$ with a Clark oxygen electrode, in a closed chamber with magnetic stirring. Mitochondria (1.5 mg protein) were pre-incubated for $3 \mathrm{~min}$ in $1.5 \mathrm{ml}$ of respiration medium containing $250 \mathrm{mM}$ sucrose, $10 \mathrm{mM}$ Hepes ( $\mathrm{pH}$ 7.2 ), $20 \mathrm{mM} \mathrm{KCl}, 5 \mathrm{mM} \mathrm{K}_{2} \mathrm{HPO}_{4}$, and $2 \mathrm{mM} \mathrm{MgCl}_{2}$ (in the absence or presence of compound) and energized with $10 \mathrm{mM}$ glutamate $/ 5 \mathrm{mM}$ malate or $10 \mathrm{mM}$ succinate. When succinate was used as respiratory substrate, the medium was supplemented with $2 \mu \mathrm{M}$ rotenone. To induce state 3 respiration, $150-200 \mu \mathrm{M}$ adenosine diphosphate (ADP) were used. FCCP-stimulated respiration (uncoupled respiration) was initiated by the addition of $1 \mu \mathrm{M} p$ - trifluoromethoxyphenylhydrazone (FCCP). The respiratory control ratio (RCR), which is calculated by the ratio between state 3 (consumption of oxygen in the presence of substrate and ADP) and state 4 (consumption of oxygen after ADP phosphorylation), is an indicator of mitochondrial membrane integrity. ADP/O ratio, which is expressed by the ratio between the amount of ADP added and the oxygen consumed during state 3 respiration, is an index of oxidative phosphorylation efficiency. Respiration rates were calculated considering that the saturation oxygen concentration was $250 \mu \mathrm{M}$ at $30^{\circ} \mathrm{C}$, and the values are expressed in percentage (\%) of control.

\subsection{Measurement of mitochondrial transmembrane potential $(\Delta \psi)$}

The mitochondrial transmembrane potential $(\Delta \psi)$ was measured indirectly based on the activity of the lipophilic cation tetraphenylphosphonium $\left(\mathrm{TPP}^{+}\right)$using a $\mathrm{TPP}^{+}$-selective electrode in combination with a $\mathrm{Ag} / \mathrm{AgCl}-$ saturated reference electrode, as previously described [23]. Mitochondria (1.5 mg protein) were incubated for $3 \mathrm{~min}$, at $30{ }^{\circ} \mathrm{C}$, in $1.5 \mathrm{ml}$ of respiratory medium [ $250 \mathrm{mM}$ sucrose, $10 \mathrm{mM}$ Hepes (pH 7.2), $20 \mathrm{mM} \mathrm{KCl}, 5 \mathrm{mM} \mathrm{K}_{2} \mathrm{HPO}_{4}$ and $2 \mathrm{mM}$ $\mathrm{MgCl}_{2}$ ] supplemented with $3 \mu \mathrm{M} \mathrm{TPP}$ (in the absence and presence of the compounds) and energized with $10 \mathrm{mM}$ glutamate $/ 5 \mathrm{mM}$ malate or $10 \mathrm{mM}$ succinate. When succinate was used as respiratory substrate the medium was supplemented with $2 \mu \mathrm{M}$ rotenone. To induce phosphorylation, 150-200 $\mu \mathrm{M}$ adenosine diphosphate (ADP) was used. No correction was made for the "passive" binding of $\mathrm{TPP}^{+}$to the mitochondria membranes because the purpose of the experiments was to show relative changes in potential rather than absolute values. As a consequence, we can anticipate some overestimation for the $\Delta \psi$ values. OSI-1210, OSI-1211 (etaftoron), and OSI-3802 did not affect $\mathrm{TPP}^{+}$binding to mitochondria membranes or the electrode response. The phosphorylation rate was calculated taking into account the time required to phosphorylate all the ADP added, and the values are expressed in percentage (\%) of control.

\subsection{Preparation of multilamellar vesicles (MLV) of dimyristoylphosphatidylcholine (DMPC)}

Aliquots from solutions of pure DMPC in $\mathrm{CHCl}_{3}$ were taken in round bottom flasks and the solvent was evaporated to dryness. The resulting lipid film was hydrated at $35^{\circ} \mathrm{C}$ (about $10^{\circ} \mathrm{C}$ above the transition temperature of the phospholipid) under $\mathrm{N}_{2}$ atmosphere, by hand shaking with an appropriate volume of $50 \mathrm{mM} \mathrm{KCl}, 10 \mathrm{mM}$ Hepes, 
$\mathrm{pH} 7.4$, to obtain a lipid concentration of $150 \mathrm{mM}$. Then, the suspension was vortexed and stabilized overnight.

\subsection{Differential scanning calorimetry (DSC)}

The 1,4-dihydropyridine derivatives OSI-1210, OSI-1211 (etaftoron), and OSI-3802 $(0,8,16$, and $30 \mu \mathrm{M})$ were added from concentrated ethanolic solutions (125 mM) to $150 \mathrm{mM}$ DMPC dispersions, to yield the compound/lipid molar ratios stated in the figures $(0,1 / 20,1 / 10$, and $1 / 5$, respectively). The lipid dispersions containing the compounds were allowed to equilibrate for $18-20 \mathrm{~h}$. After, they were hermetically sealed in aluminium pans and thermograms were recorded in a PerkinElmer Pyris 1 differential scanning calorimeter. The DSC measurements were performed at a scan rate of $5{ }^{\circ} \mathrm{C} / \mathrm{min}$, over a temperature range from 20 to $60^{\circ} \mathrm{C}$. This rate yielded thermograms essentially identical to those obtained at $1^{\circ} \mathrm{C} / \mathrm{min}$, meaning that thermodynamic equilibrium is maintained at the scanning rate of $5{ }^{\circ} \mathrm{C} / \mathrm{min}$. Data acquisition and analysis were performed using the software provided by PerkinElmer. To check for the reproducibility of the results, three different samples were scanned for each compound/lipid ratio. For each sample, three heating and two cooling scans were recorded. The onset and completion of the phase transition were determined from the intersections of the peak slopes with the baseline of the thermograms [24]. Cooling scans yielded thermograms very similar to the heating scans, but, according to the literature [25], the transitions in cooling curves are shifted, by about $1{ }^{\circ} \mathrm{C}$, to lower temperatures. Therefore, due to the supercooling phenomenon, accurate thermotropic transitions are evaluated from heating curves. Consequently, heating scans have been used throughout this study. At the end of the experiments, the aluminium pans were opened and the samples dissolved in chloroform/methanol (5/1) mixtures. The phospholipids content was determined by measuring inorganic phosphate [26] after hydrolysis of the extracts at $180^{\circ} \mathrm{C}$ in $70 \%$ $\mathrm{HClO}_{4}[27]$.

\subsection{Statistical analysis}

All the experiments were performed using four independent experiments with different mitochondrial preparations. The values are expressed as mean \pm S.E. Means were compared using one-way ANOVA test, followed by the post hoc Tukey test. Statistical significance was set at $p<0.05$.

\subsection{Chemicals}

All chemicals were obtained from Sigma Chemical Company (St. Louis, MO, USA) except OSI-1210, OSI-1211 (etaftoron), OSI-3802, and OSI-1212 (cerebrocrast) which were synthesized at the Latvian Institute of Organic Synthesis, 21 Aizkraukles Street, Riga LV-1006. All the compounds tested were dissolved in absolute ethanol. Pure solutions of absolute ethanol were added to controls, with the highest volume used of each compound ethanol solutions [0.1\% $(\mathrm{v} / \mathrm{v})$ of the experiments final volume], and had no effects on the measured activities.

\section{Results}

3.1. Effects of OSI-1210, OSI-1211 (etaftoron), and
OSI-3802 on rat liver mitochondrial bioenergetics

For comparative analysis, we studied the effects of OSI-1210, OSI-1211 (etaftoron), and OSI-3802 on glutamate/malate- and succinate-supported respiratory rates of states 3, 4 and FCCP-stimulated respiration (uncoupled respiration) of rat liver mitochondria (Fig. 2). State 3 respiration and uncoupled respirations were depressed by these compounds for concentrations of up to $500 \mu \mathrm{M}$, whereas state 4 respiration was stimulated, using either glutamate/malate or succinate as respiratory substrates. However, a higher stimulatory effect on state 4 was induced by OSI-3802 as compared with OSI-1210 and OSI-1211 (etaftoron). In fact, OSI-3802 maximizes state 4 stimulation $(\sim 100 \%)$ at a concentration of $100 \mu \mathrm{M}$, whereas low graduate effects $(\sim 45 \%)$ were detected with OSI-1210 and OSI-1211 (etaftoron) for concentrations of up to $500 \mu \mathrm{M}$. According to these data, OSI-3802 is the compound that most affected inner mitochondrial membrane permeability to cations. For each compound tested, state 3 respiration was depressed at identical levels of uncoupled respiration. However, at $500 \mu \mathrm{M}$, higher inhibitory effects ( 25\%) were induced by OSI-1211 (etaftoron) and OSI-3802 when compared with OSI-1210 ( 10\%). According to these data, the inhibitory effect on state 3 , being similar to the effect on uncoupled respiration, is mainly due to interference of the compounds with the mitochondrial respiratory chain rather than on some specific oxidative phosphorylation intervener. The more discrete effects of the compounds on malate/glutamate-supported respiration when compared with succinate-supported respiration (Figs. 2-4) are not due to a lower inhibitory effect of the compounds on complex I than on complex II because the depression of uncoupled respiration was identical using either glutamate/malate or succinate as substrate. Hence, the reason why complex I is more protected against the effects of these compounds than complex II remains to be clarified.

The effects of OSI-1210, OSI-1211 (etaftoron), and OSI-3802 on glutamate/malate- and succinate-dependent respiratory indices, namely respiratory control (RCR), and $\mathrm{ADP} / \mathrm{O}$ ratios of rat liver mitochondria, are shown in Fig. 3. All the compounds depressed the RCR and ADP/O, using either glutamate/malate or succinate as respiratory substrates. However, differently from OSI-3802, which decreased RCR and ADP/O at a higher degree (almost maximum at $100 \mu \mathrm{M}$ ), lower graduate effects were detected with OSI-1210 and OSI-1211 (etaftoron) for concentrations up to $500 \mu \mathrm{M}$, with slightly higher efficiency for OSI-1211 (etaftoron). The fact that OSI-3802 $(500 \mu \mathrm{M})$ stimulates glutamate/malate- and succinate-dependent state 4 respiration at a higher level (100\%) than OSI-1211 (etaftoron) (40\%) with a similar effect on $\mathrm{ADP} / \mathrm{O}$ ratio suggests that the last compound has putatively mixed effects, inducing lower membrane permeability to cations than OSI-3802 and slightly higher inhibition on the respiratory chain.

To confirm these observations, we tested the effects of these compounds on glutamate/malate- and succinatedependent phosphorylation rate (Fig. 4). OSI-3802 was the 
(A) Glutamate/Malate

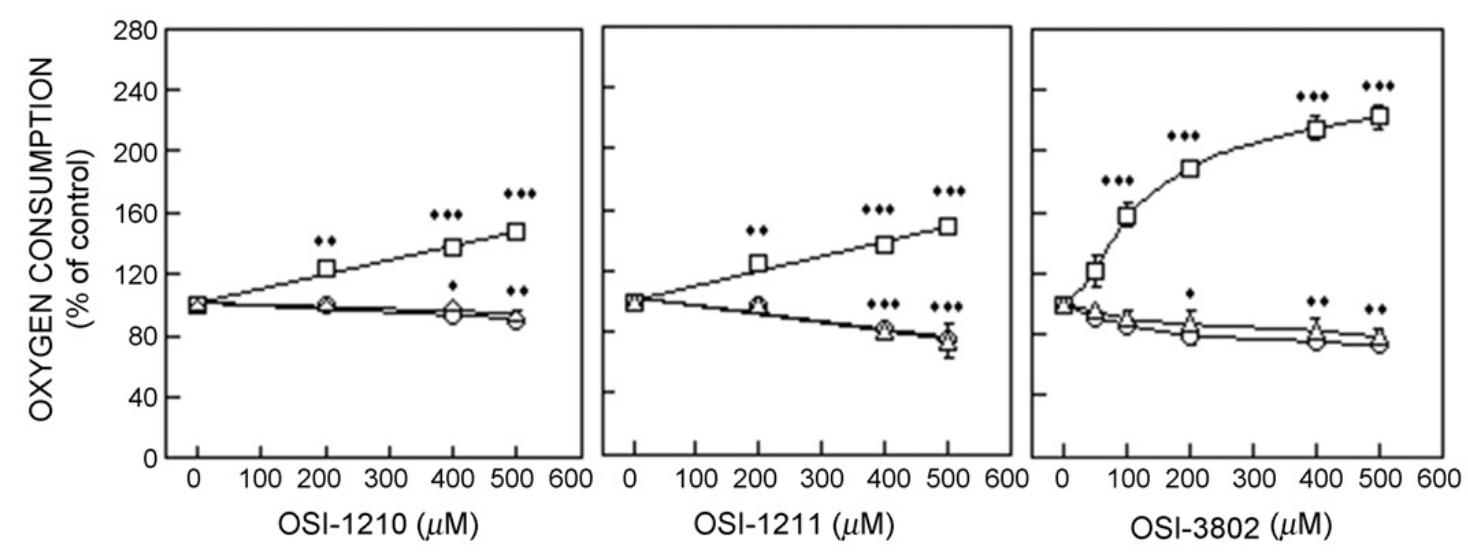

(B) Succinate
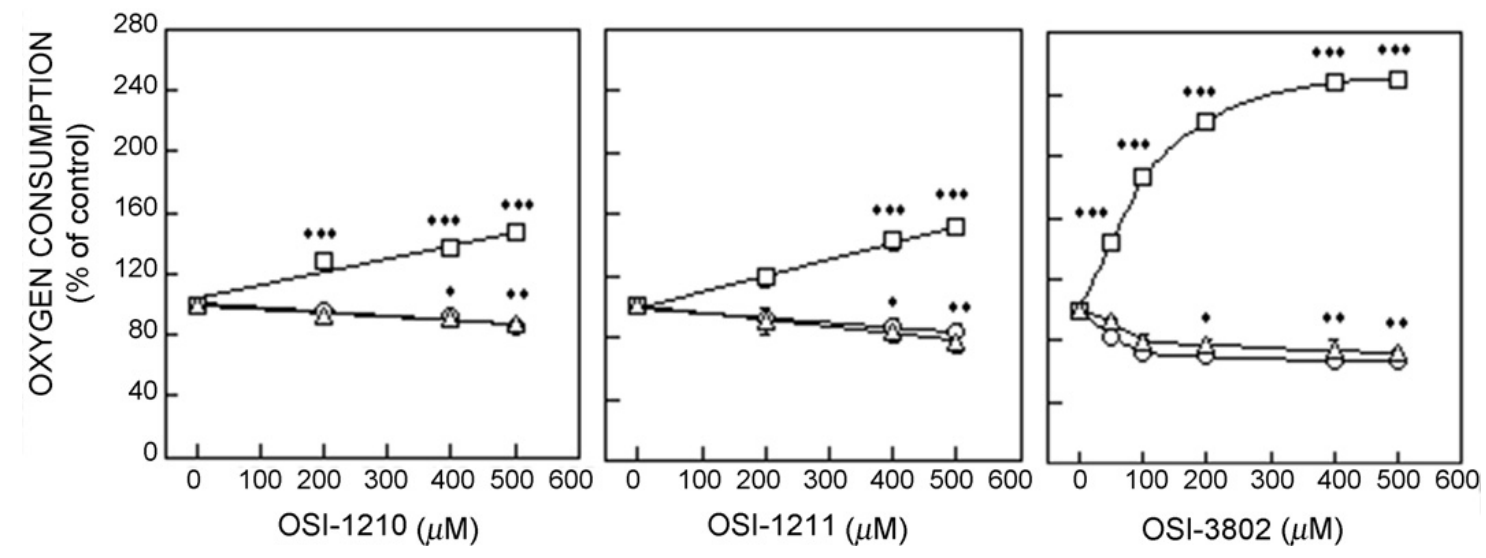

Fig. 2. Effects of OSI-1210, OSI-1211 (etaftoron), and OSI-3802 on glutamate/malate-supported (A) and succinate-supported respiration (B). $\mathrm{O}_{2}$ consumption in state 3 (circles), state 4 (squares) and FCCP-uncoupled respiration (triangles) of rat liver mitochondria. Mitochondria were incubated in reaction medium, at conditions described in Section 2. Control values expressed in $\mathrm{nmol} \mathrm{O}_{2} \mathrm{mg}^{-1}$ protein $\mathrm{min}^{-1}$ : (A) state $3=35.2 \pm 1.60$, state $4=6.5 \pm 0.370, \mathrm{FCCP}=76 \pm 9.5 ;(B)$ state $3=46.67 \pm 2.41$, state $4=11 \pm 0.48$, FCCP $=81.06 \pm 3.37$. Results correspond to the mean \pm S.E. of four experiments obtained from different mitochondrial preparations (when the error bars are not visible, S.E. is encompassed by the size of the symbols). ${ }^{*} p<0.05 ;{ }^{* *} p<0.01$; ${ }^{* * *} p<0.001$ compared with the control (without DHP compounds).

most potent compound affecting these parameters, with higher efficiency when succinate was the respiratory substrate. As for $\mathrm{O}_{2}$ consumption results, these effects were also maximized at $100 \mu \mathrm{M}$ OSI-3802, whereas lower graduate effects were recorded for the other two compounds. These effects of OSI-3802 should putatively be due to its ability to interfere with the mitochondrial inner membrane permeabilization to cations, rather than to interference with the mitochondrial respiratory chain, in agreement with its effects on state 4 and uncoupled respirations, respectively (Fig. 2). Thus, to confirm this hypothesis alterations on membrane physical properties induced by the compounds were tested.

\subsection{Effects of OSI-1210, OSI-1211 (etaftoron), and OSI-3802 on membrane physical properties}

The DSC heating scans of multilamellar vesicles (MLV) of DMPC incorporating increasing concentrations of the compounds under study [OSI-1210, OSI-1211 (etaftoron), OSI-3802) and OSI-1212 (cerebrocrast) (used for comparison)], in the range of $0.05(1 / 20)$ to $0.2(1 / 5)$ compound/DMPC molar ratio, are shown in Fig. 5A. The corresponding molar ratio dependence of the thermotropic parameters, the transition temperature (Tm) and the transition enthalpy, $\Delta H(\mathrm{~J} / \mathrm{g})$, are given in Fig. 5B1, B2, and C. Although the compounds have been added from concentrated ethanolic solutions, the volume of ethanol (a membrane fluidizer) in membrane preparations was somewhat appreciable. Therefore, for each series of assays with the compounds at the same molar ratio, a preparation of DMPC liposomes containing the same volume of ethanol was used as a control (Fig. 5A). In our experimental conditions, increasing volumes of ethanol only slightly affect the $\mathrm{Tm}$ and $\Delta H$ of DMPC endotherms, as we can appreciate by the standard deviations of the media for both parameters in the control conditions (drug/DMPC molar ratio of zero), represented in Fig. 5B1, B2, and C.

Drug-free DMPC dispersions (ethanol containing MLV) show a sharp endotherm, centered at $24.21 \pm 0.13^{\circ} \mathrm{C}$, with a $\Delta H$ value $(41.08 \pm 0.59 \mathrm{~J} / \mathrm{g})$ characteristic of DMPC bilayers. All the compounds tested induced a shift of DMPC main phase transition to lower temperatures and a broadening of the endothermic profile (Fig. 5A). However, OSI-3802 and its analogue OSI-1212 (cerebrocrast) were the compounds that most affected DSC derived thermodynamic parameters, as shown in Fig. 5B2 and C. Thus, whereas OSI1210 and OSI-1211 (etaftoron) did not significantly affect 
(A) Glutamate/Malate
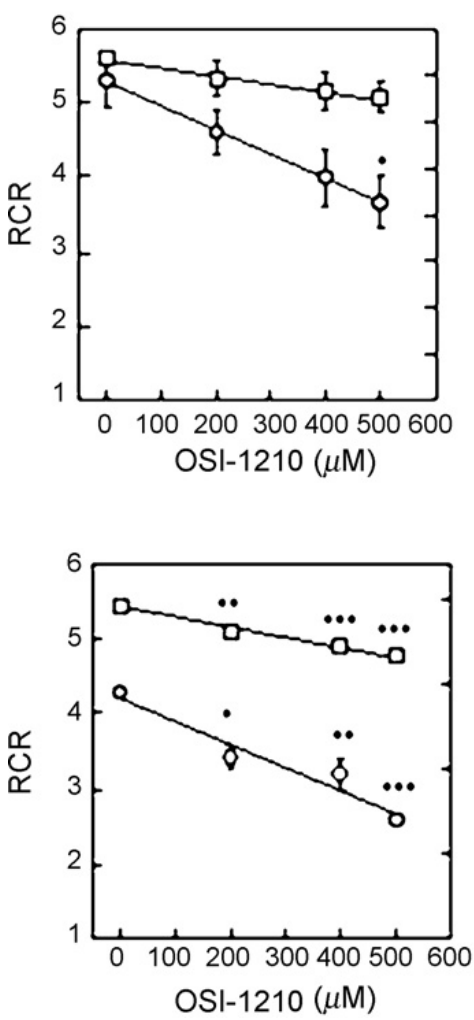

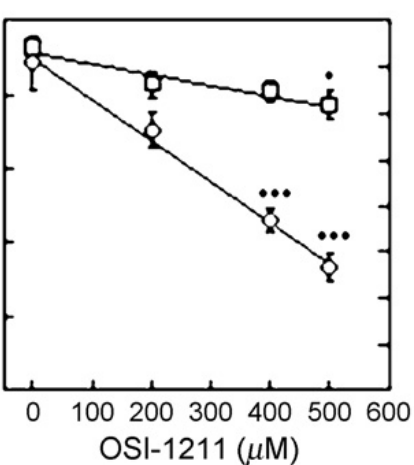

(B) Succinate

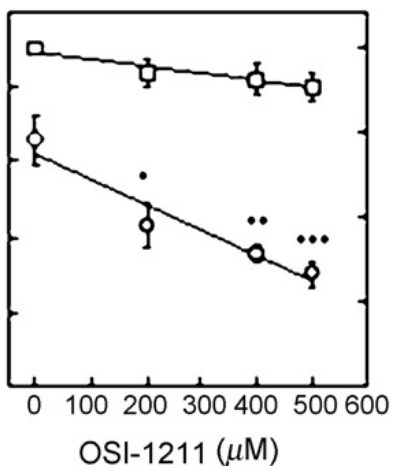

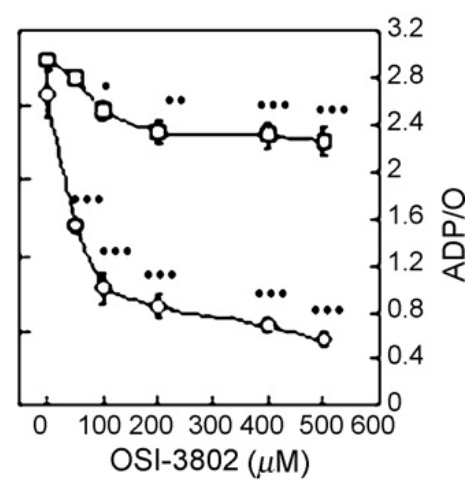

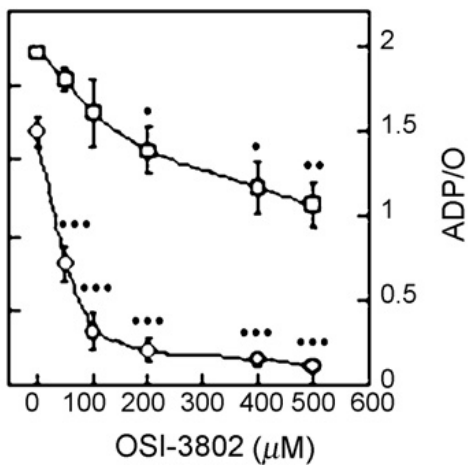

Fig. 3. Effects of OSI-1210, OSI-1211 (etaftoron), and OSI-3802 on glutamate/malate-supported (A) and succinate-supported (B) respiration indices: respiratory control ratio (RCR) (open circles) and ADP/O (open squares) of rat liver mitochondria. Mitochondria were incubated in reaction medium, at conditions described in Section 2. Results correspond to the mean \pm S.E. of four experiments obtained from different mitochondrial preparations (when the error bars are not visible, S.E. is encompassed by the size of the symbols). ${ }^{*} p<0.05{ }^{* *} p<0.01 ;{ }^{* * *} p<0.001$ compared with the control (in the absence of compounds).

DMPC phase transition temperature range (Fig. 5B1) and induce a slight decrease of $\Delta H$ (Fig. 5C), OSI-3802 and OSI-1212 (cerebrocrast) promote a significant shift of the endotherm to lower temperatures (specially affecting the onset temperature; Fig. 5B2) and a pronounced decrease of $\Delta H$ (Fig. 5C). Analysis of the partial phase diagrams shown in Fig. 5B1 and B2, obtained by plotting the onset and end temperatures of the phase transition of drug/DMPC systems as a function of the drug/lipid molar fractions represented in Fig. 5A, leads to the conclusion that the broadening induced by OSI-3802 and OSI-1212 (cerebrocrast) in the phase transition of DMPC bilayers resulted from a particu-
(A) Glutamate/Malate

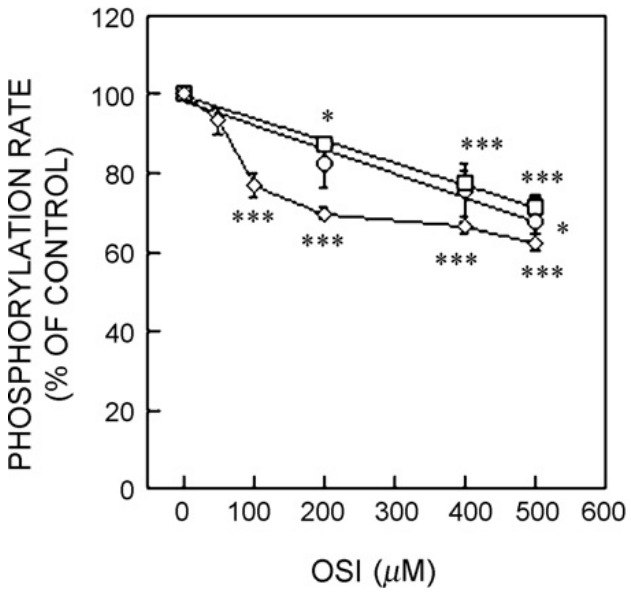

(B) Succinate

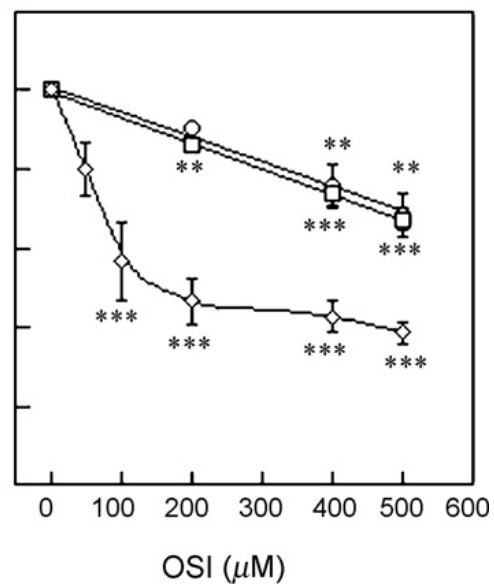

Fig.4. Effects of OSI-1210 (circles), OSI-1211 (etaftoron) (squares), and OSI-3802 (triangles) on glutamate/malate-supported (A) and succinate-supported (B) phosphorylation. The phosphorylation rate was calculated taking into account the time required to phosphorylate all the ADP added by using a TPP ${ }^{+}$-selective electrode (as described in Section 2). Control values expressed in nmol ADP mg ${ }^{-1}$ protein min ${ }^{-1}$ : (A) $197 \pm 12.016$ and (B) $178 \pm 13.25$. Results correspond to the mean \pm S.E. of four experiments obtained from different mitochondrial preparations (when the error bars are not visible, S.E. is encompassed by the size of the symbols). ${ }^{*} p<0.05 ;{ }^{* *} p<0.01 ;{ }^{* * *} p<0.001$ compared with the control (in the absence of compounds). 
larly abrupt decrease of the temperatures of the solidus line, since the temperature change in fluidus line is softer. The broadening of the transition reflects a decrease in the size of the cooperative unit that actually undergoes the phase transition. This effect is consistent with the concentrationdependent decrease of $\Delta H$, which may suggest that an increasing fraction of lipids become unable to participate in the thermal phase transition. These events point to a

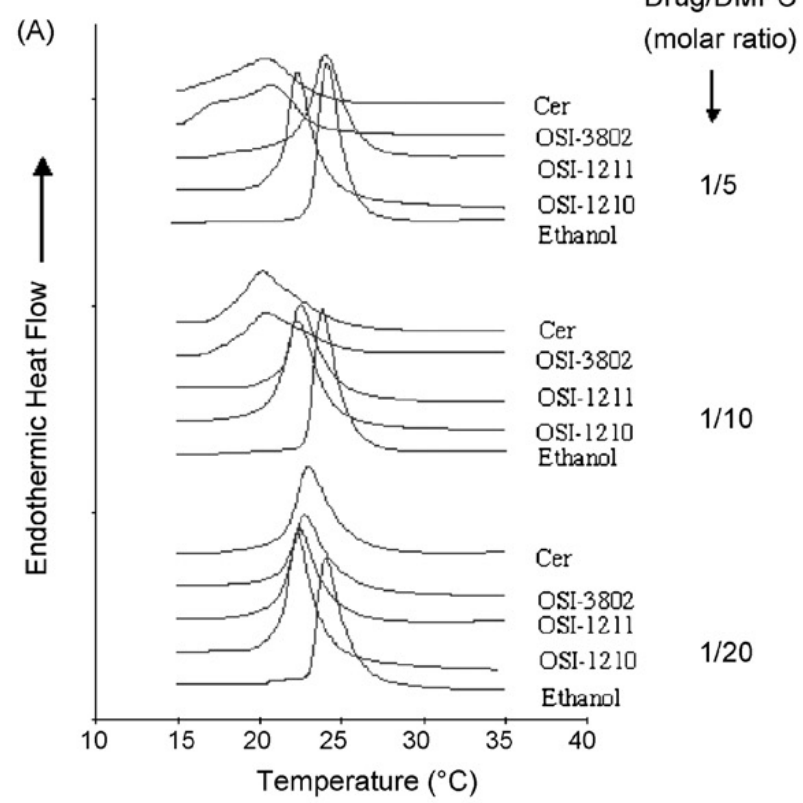

(B1)

(B2)
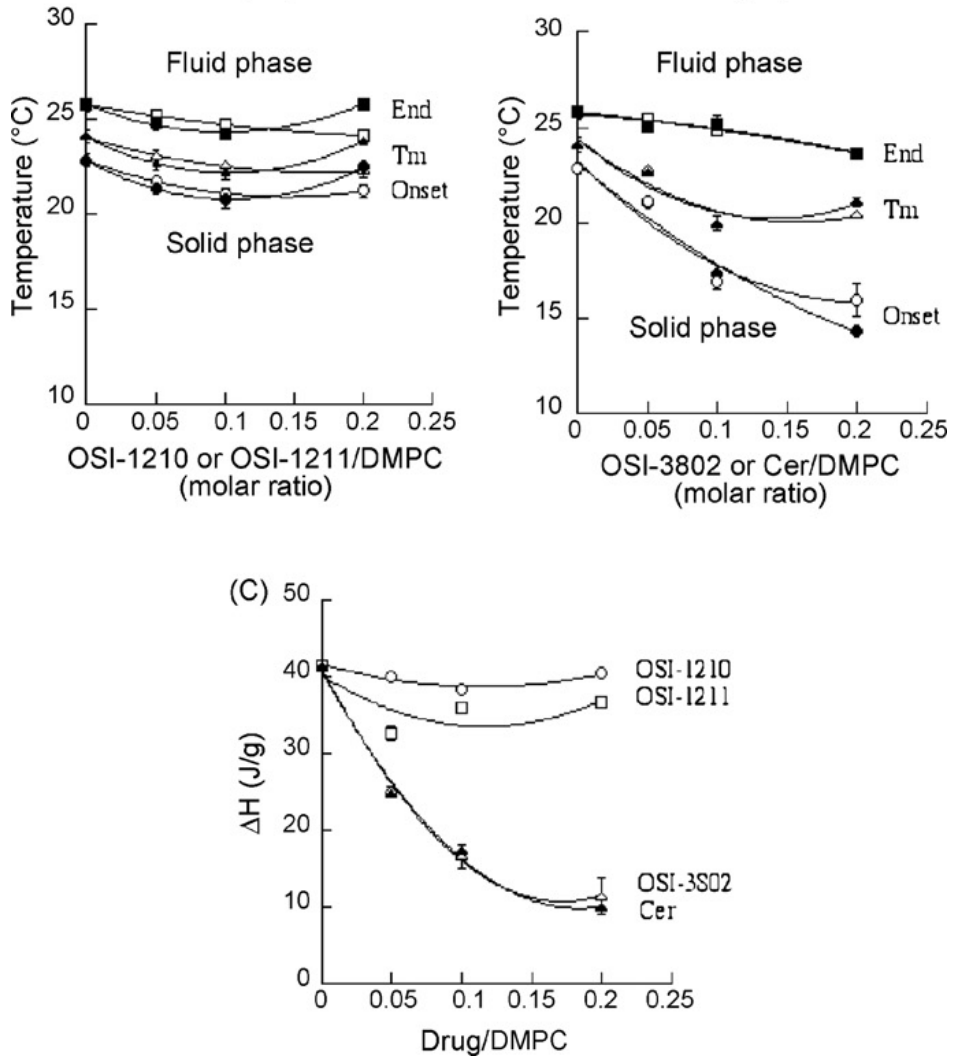

Fig. 5. Effects of OSI-1210, OSI-1211 (etaftoron), OSI-3802 and cerebrocrast on differential scanning calorimetry heating thermograms of DMPC membrane bilayers. The drug/lipid molar ratios were successively increased from $1 / 20$ to $1 / 5$, and they are indicated on the curves. The scans represent the average of two trials consisting of four samples for each drug concentration (A). Thermal phase diagram for mixtures of DMPC and drugs (B1 and B2) was built with data from the thermograms. Open symbols correspond to OSI-1210 and OSI-3802, and closed symbols correspond to OSI-1211 and cerebrocrast (Cer). Onset, Tm and End, correspond to the onset, midpoint and completation temperature of the transition, respectively. Enthalpy change for the thermotropic profile of DMPC membrane bilayers, as a function of drug/lipid molar ratio (C). Each point represents the average of four independent measurements (vertical bars indicate \pm S.E.). When the error bars are not visible, S.E. is encompassed by the size of the symbols. 


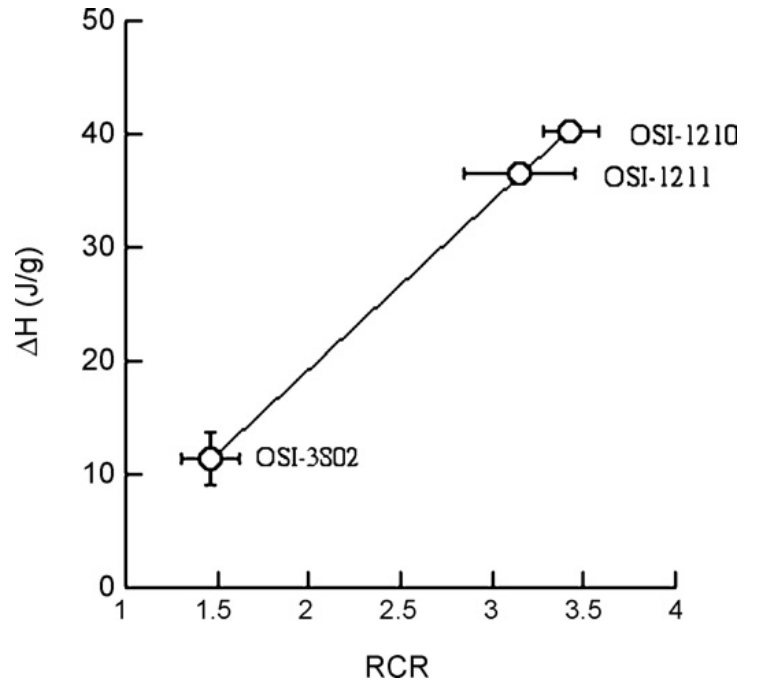

Fig. 6. Relationship between the effects of $200 \mu \mathrm{M}$ OSI-1210, OSI-1211 (etaftoron), and OSI-3802 on succinate-dependent respiratory control ratio (RCR) of rat liver mitochondria and the enthalpy change $(\Delta H)$ for the thermotropic profile of DMPC membrane bilayers, at 0.2 drug/DMPC molar ratio. When the error bars are not visible, S.E. is encompassed by the size of the symbols. The equation for the linear fit has the form $y=-10.103+14.738 x$ with a correlation coefficient, $R=0.99996$. Vertical and horizontal lines on the symbols correspond to the error bars of RCR and $\Delta H$, respectively. When the error bars are not visible, S.E. is encompassed by the size of the symbols.

severe perturbation of the membrane lipid organization induced by OSI-3802 and OSI-1212 (cerebrocrast). On the basis of these studies, the compounds OSI-1210 and OSI1211 (etaftoron) seem to be much more weaker disturbers of membrane physical properties.

\subsection{Correlation between the effects of OSI-1210, OSI-1211 (etaftoron), and OSI-3802 on rat liver mitochondrial bioenergetics and membrane physical properties}

Considering the RCR of rat liver mitochondria and the $\Delta H$ for the thermotropic events of DMPC bilayers as indicators of mitochondrial membrane integrity and membrane lipid organization, respectively, a correlation between those parameters was tentatively established. The values of succinate-dependent RCR obtained with $200 \mu \mathrm{M}$ OSI-1210, OSI-1211 (etaftoron), and OSI-3802 were plotted versus the values of $\Delta H$ of the DMPC endotherms in the presence of the correspondent compounds at a 0.2 drug/lipid molar ratio, allowing to obtain a correlation of 0.999 (Fig. 6). Although the effects of the compounds on malate/glutamate-supported respiration were more discrete than on succinate-supported respiration (Figs. 2-4), a good correlation between the values of glutamate/malatedependent RCR and the values of $\triangle H$ of the DMPC endotherms in the presence of the correspondent compounds was also observed (results not shown). This good correlation suggests that the effects on mitochondrial function are putatively related to compounds-induced alterations of membrane structure and dynamics. This hypothesis is based on the similar dependence of the physical and bioenergetics parameters on the length of the alkoxyl chain in position 3 and 5 of the DHP ring. It was pre- dictable that compounds with a longer lateral chain induce stronger perturbation in the membrane lipid organization and, therefore, membrane-dependent organelle functions.

\section{Discussion}

This study was designed to evaluate the effects of OSI1210, OSI-1211 (etaftoron), and OSI-3802 on mitochondrial bioenergetics and membrane physical properties, as an approach to correlate the biological activities of those compounds and a particular characteristic of their molecular structure, the length of the alkoxyl chain in positions 3 and 5 of the DHP.

Mitochondrial bioenergetics was assessed by evaluating several mitochondrial parameters from the respiratory chain (states 3, 4, and FCCP-stimulated respiration, respiratory control ratio ( $\mathrm{RCR}$ ) and $\mathrm{ADP} / \mathrm{O}$ ratio) and oxidative phosphorylation system (phosphorylation rate) using both glutamate/malate and succinate as respiratory substrates. According to the mitochondrial parameters affected it is possible to conclude how a membrane-active agent interferes with mitochondrial bioenergetics: by perturbing the permeability (integrity) of the inner mitochondrial membrane (state 4 respiration); by impairing the respiratory chain (FCCP-stimulated respiration); and/or by acting at the level of the phosphorylation system (state 3 respiration). Consequently, these data may be useful to establish the potential pharmacological and/or toxicological action mechanism of a compound at the mitochondrial level. As the compounds we used are lipophilic molecules, with a predictable strong partitioning in membranes, parallel biophysics studies (DSC) were performed using DMPC membrane bilayers incorporating increasing concentrations of the compounds under study.

It was observed that all the compounds tested depressed glutamate/malate- and succinate-dependent state 3 and uncoupled respiration concomitantly with stimulation of state 4 respiration (Fig. 2). Respiratory indices RCR and ADP/O ratio (Fig. 3) and phosphorylation rate (Fig. 4) were also depressed. According to these data, the compounds under study interfere with rat liver mitochondrial bioenergetics by affecting the mitochondrial respiratory chain and the inner mitochondrial membrane permeability to cations. However, the most striking effects were observed with OSI-3802, the compound with the longest chain in positions 3 and 5 of the DHP ring. The compounds with the shortest and intermediate chains, OSI-1210 and OSI1211 (etaftoron), respectively, had similar weak effects (Figs. 2-4). However RCR was more affected by OSI-1211 (etaftoron) than OSI-1210. According to these results, the presence of three carbon atoms in the ester moiety in positions 3 and 5 of the DHP structure is an essential molecular characteristic to determine the interference of these compounds with mitochondrial bioenergetics. This assumption is supported by comparing the results obtained in this study (Figs. 2-4) with the results of identical experiments previously performed with OSI-1212 (cerebrocrast) [16], clearly showing that, at high concentrations $(>25 \mu \mathrm{M})$, like its isomer OSI-3802, has a significant effect on mitochondrial bioenergetics by affecting inner mitochondrial membrane permeability to cations. The influence of 3,5- 
dialkoxycarbonyl chain length of the 1,4-dihydropyridines on mitochondrial bioenergetics was previously reported for other DHP derivatives. Thus, in the case of 4unsubstituted 2,6-dimethyl-1,4-dihydropyridines, elongation of the alkyl chain in the positions 3 and 5 by introduction of 3,5-dibutoxycarbonyl- or 3,5-diamyloxycarbonylesters instead of 3,5-diethoxycarbonyl-ester (namely in the case of Hantzsch compound-diludin, ethidin or diethone), increase the uncoupling ability both in normal liver cell mitochondria and in solid tumor cell mitochondria, as observed by RCR and ADP/O decrease [28].

The hypothesis that the introduction of three carbon atoms in the ester moiety in positions 3 and 5 of the DHP structure is essential to the disturbing action on bioenergetics with the compounds under study was further supported by the finding that OSI-3802, like its analogue OSI-1212 (cerebrocrast), was the most potent compound promoting perturbations on the lipid membrane organization, as clearly illustrated by DSC data of DMPC model membranes (Fig. 5 and [17]). According to the respiratory data, the compounds OSI-1210 and OSI-1211 (etaftoron) also induced similar weak perturbations on DMPC thermotropic behaviour (Fig. 5). The strong correlation observed between the effects induced by OSI-1210, OSI-1211 (etaftoron), and OSI-3802 on succinate-dependent RCR of rat liver mitochondria and on $\triangle H$ of DMPC membrane bilayers endothermic (Fig. 6), Which suggests a putative relationship between mitochondrial membrane integrity and membrane lipid organization, clearly indicates the dependence of these events on the length of the alkoxyl chain in positions 3 and 5 of the DHP ring.

In terms of the effects on DMPC membrane bilayers, it is not surprising the relationship between those effects and the structural particularities of the compounds under study. It was expected that the increased length of the lateral chains of the DHP ring in OSI-3802 and OSI-1212 (cerebrocrast) as compared with the other compounds, contribute to a stronger perturbation of DMPC acyl chains packing in the gel phase, as was confirmed by the abrupt decrease of the temperatures of the solidus line with the increase of both OSI-3802, and OSI-1212 (cerebrocrast)/DMPC molar ratio (Fig. 5B2). Complementary information would be obtained by measuring order parameters by the use of fluorescent fluidity probes. However, these studies could not be carried out because of the fluorescence quenching of probes in the presence of these compounds. On the other hand, the broadening of the endothermic transition (Fig. 5A) and the decrease of $\Delta H$ (Fig. 5C) induced by OSI-3802 are also consistent with the formation of heterogeneous lipid domains differently enriched in the compound and thus showing different physical properties. Assuming the effects observed in our model membranes may be extrapolated to other membrane systems, namely mitochondrial membranes, and based on the knowledge that membrane physical properties are critical for most membrane activities, including permeability, and protein-protein interactions, it is predictably that the compounds which most affect membrane lipid organization and packing have the higher impact on membrane-dependent mitochondrial functions. This hypothesis could be reinforced if compounds physical effects could be evaluated directly in intact mitochondria. Calorimetric studies, however, are difficult as consequence of the interference of transitions due to protein denaturation.

Although contradictory "in vitro" and "in vivo" results were reported concerning the pharmacological effectiveness of OSI-1212 (cerebrocrast) and OSI-1211 (etaftoron) [5-7], our experimental data, obtained with high concentrations of the compounds ( $200 \mu \mathrm{M}$ for mitochondria, and $0.2 \mathrm{drug} / \mathrm{lipid}$ molar ratio for DMPC), are in good line with the suggestion that the differences on the pharmacological effectiveness of these compounds, at low concentrations $\left(10^{-7}\right.$ down to $\left.10^{-9} \mathrm{M}\right)$ "in vitro" and at low doses $(0.5$ and $1.5 \mathrm{mg} \mathrm{kg}^{-1}$ ) "in vivo", are related to the number of carbon atoms in the ester moiety in positions 3 and 5 of the DHP structure $[5,6]$. On the other hand, the similarity of the effects induced by OSI-1212 (cerebrocrast) and its isomer OSI-3802 observed in our study on both bioenergetics and biophysical parameters, indicates that differences on the pharmacological effectiveness of these compounds, reported at low doses ( 0.5 and $1.5 \mathrm{mg} \mathrm{kg}^{-1}$ ) "in vivo" [6], may be due to factors other than the length of the alkoxyl chain in positions 3 and 5 of the DHP ring.

In conclusion, from the tested compounds (OSI-1210, OSI-1211 (etaftoron), and OSI-3802), OSI-3802 was the most efficient in affecting mitochondrial bioenergetics and the physical properties of DMPC membrane bilayers. According to these results, the presence of three carbon atoms in the ester moiety in positions 3 and 5 of the DHP structure is essential to the specific action of these compounds at the mitochondrial and the lipid bilayer level. Independently of these effects to be or not to be involved in the toxicological and pharmacological actions of the compounds, our study provides convincing data pointing out the relevance of the number of carbon atoms in the ester moiety in positions 3 and 5 of the DHP structure for the biological activity of these 1,4-dihydropyridines.

\section{Acknowledgements}

This study was supported by grants of the Portuguese Research Council, Environment and Life Science Institute, Institute of Marine Research, and Center for Neuroscience and Cell Biology of the University of Coimbra.

\section{References}

[1] D.J. Triggle, The 1,4-dihydropyridine nucleus: a pharmacophoric template. Part 1. Actions at ion channels, Mini Rev. Med. Chem. 3 (2003) 215-223.

[2] R.P. Mason, P. Marche, T.H. Hintze, Novel vascular biology of thirdgeneration L-type calcium channel antagonists: ancillary actions of amlodipine, Arterioscler. Thromb. Vasc. Biol. 23 (2003) 2155-2163.

[3] Sh. Yamagishi, K. Nakamura, K. Takenaka, T. Matsui, H. Inoue, Pleiotropic effects of nifedipine on atherosclerosis, Curr. Pharm. Design 12 (2006) 1543-1547.

[4] G. Duburs, B. Vigante, A. Plotniece, A. Krauze, A. Sobolevs, J. Briede, V. Klusa, A. Velena, Dihydropyridine derivatives as bioprotectors. Chim. Oggi/Chem. Today, in press.

[5] J. Briede, D. Daija, E. Bisenieks, N. Makarova, J. Uldrikis, J. Poikãns, G. Duburs, Effects of some 1,4-dihydropyridine Ca antagonists on the blast transformation of rat spleen lymphocytes, Cell Biochem. Funct. 17 (1999) 97-105.

[6] A. Vaitkuviene, A. Ulinskaite, R. Meskys, G. Duburs, V. Klusa, E. Liutkevicius, Study of the interaction of 1,4-dihydropyridine derivatives 
with glucocorticoid receptors from the rat liver, Pharmacol. Rep. 58 (2006) 551-558.

[7] J. Briede, M. Stivrina, D. Stoldere, E. Bisenieks, J. Uldrikis, J. Poikãns, N. Makarova, G. Duburs, Effect of new and known 1,4-dihydropyridine derivatives on blood glucose levels in normal and streptozotocininduced diabetic rats, Cell Biochem. Funct. 22 (2004) 219-224.

[8] T.M. Plotnikova, M.B. Plotnikov, G. Duburs, E.A. Bisenieks, J.R. Uldrikis, N.V. Makarova, Agent decreasing hemoglobin affinity to oxygen, Patent RU 2,061,479 (1996).

[9] C. Luxo, A.S. Jurado, V.M.C. Madeira, Toxicity assessment of tamoxifen by means of a bacterial model, Appl. Biochem. Biotechnol. 87 (2000) 219-232.

[10] R.A. Videira, M.C. Antunes-Madeira, V.M.C. Madeira, Ethylazinphos interaction with membrane lipid organization induces increase of proton, Toxicol. Appl. Pharmacol. 175 (2001) 209216.

[11] J.B.A. Custódio, L.M. Almeida, V.M.C. Madeira, The anticancer drug tamoxifen induces changes in the physical properties of model and native membranes, Biochem. Biophys. Acta 1150 (1993) 123-129.

[12] R. Scatena, P. Bottoni, G. Botta, G.E. Martorana, B. Giardina, The role of mitochondria in pharmacotoxicology: a reevaluation of an old, newly emerging topic, Am. J. Physiol. Cell Physiol. 293 (2007) C12-C21.

[13] A.H. Velena, G.J. Dubur, R.O. Vitolina, A.A. Kimenis, M.J. Selga, G.V. Zarinsh, E.V. Narusevicius, R.A. Macianskiene, V.I. Gendviliene, B.Z. Simkhovich, Effect of ryodipine on electrochemical parameters of heart and vessels, cAMP phosphodiesterase activity and swellingcontraction cycle of mitochondria, Arzneim. -Forsch./Drug Res. 35 (1985) 907-914.

[14] R.O. Vitolina, D.A. Berzina, A.H. Velena, I.A. Vucina, A.A. Kimenis, G. Duburs, Protective action of the calcium antagonist foridon in acute myocardial ischemia, Cardiology 27 (1987) 90-93 [in Russian].

[15] A. Velena, A. Skujins, S. Svirskis, E. Bisenieks, J. Uldrikis, J. Poikans, G. Duburs, V. Klusa, Modification of swelling-contraction-aggregation processes in rat muscle mitochondria by the 1,4-dihydropyridines, cerebrocrast und glutapyrone, themselves and in the presence of azidothymidine, Cell Biochem. Funct. 15 (1997) 211-220.

[16] M.A.S. Fernandes, M.S. Santos, J.A.F. Vicente, A.J.M. Moreno, A. Velena, G. Duburs, C.R. Oliveira, Effects of 1,4-dihydropyridine derivatives (cerebrocrast, gammapyrone, glutapyrone, and diethone) on mitochondrial bioenergetics and oxidative stress: a comparative study, Mitochondrion 3 (2003) 47-59.
[17] M.A.S. Fernandes, A.S. Jurado, R.A. Videira, M.S. Santos, A.J.M. Moreno, A. Velena, G. Duburs, C.R. Oliveira, J.A.F. Vicente, Cerebrocrast promotes the cotransport of $\mathrm{H}^{+}$and $\mathrm{Cl}^{-}$in rat liver mitochondria, Mitochondrion 5 (2005) 341-351.

[18] V. Klusa, Cerebrocrast, a cognition enhancer and neuroprotectant: focus on mitochondrial functions, Auton. Autacoid Pharmacol. 26 (2006) 60-62.

[19] J.A.F. Vicente, G. Duburs, V. Klusa, J. Brieda, L. Klimaviciusa, A. Zharkovsky, M.A.S. Fernandes, in: A.J.M. Moreno, P.J. Oliveira, C.M. Palmeira (Eds.), Mitochondrial Pharmacology and Toxicology, Transworld Research Network, Kerala, 2006, pp. 185-197.

[20] G.V. Belevitch, G.Ya. Dubur, G.E. Dobretsov, N.K. Kurek, M.M. Spirin, Calcium antagonists, riodipine, nifedipine and verapamil, binding to model and biological membranes: fluorescence analysis, Biol. Membr. 5 (1988) 768-776 (in Russian).

[21] P. Gazotti, K. Malmstron, M.A. Crompton, in: E. Carafoli, G. Semenza (Eds.), Membrane Biochemistry, Springer Verlag, New York, 1979, pp. 62-69.

[22] G. Gornall, C.J. Bardawill, M.M. David, Determination of serum proteins by means of the biuret reaction, J. Biol. Chem. 177 (1949) 751-766.

[23] N. Kamo, M. Muratsugu, R. Hongoh, N. Kobatake, Membrane potential of mitochondria measured with an electrode sensitive to tetraphenylphosphonium and relationship between proton electrochemical potential and phosphorylation potential in steady state, J. Membr. Biol. 49 (1979) 105-121.

[24] R.D. Koynova, A.I. Boyanov, B.G. Tenchov, On the phase diagram of an L-dipalmitoylphosphatidylcholine/cholesterol mixture, FEBS Lett. 187 (1985) 65-68.

[25] P.W.M. Van Dijck, B. De Kruijff, L.L.M. Van Deenen, J. De Gier, R.A Demel, The presence of cholesterol for phosphatidylcholine in mixed phosphatidylcholine-phosphatidylethanolamine bilayers, Biochem. Biophys. Acta 455 (1976) 576-587.

[26] G.R. Bartlett, Phosphorus assay in column chromatography, J. Biol. Chem. 234 (1959) 466-468.

[27] C.J.F. Böttcher, C.M. Van Gent, C. Pires, A rapid and sensitive sub-micro phosphorus determination, Anal. Chim. Acta 24 (1961) 203-204.

[28] K.K. Kumsar, A.Kh. Velena, G.Ia. Dubur, Ia.R. Uldrikis, A.A. Zidermane, Effect of 1,4-dihydropyridine and 1,6-dihydropyrimidine derivatives on the energy metabolism of hepatic and tumor cells, Bookhimia 36 (1971) 1204-1209 (in Russian). 